\title{
BIOMETRIA DA SINÚSIA ARBÓREA DE FRAGMENTOS FLORESTAIS DO DOMÍNIO TROPICAL ATLÂNTICO (ILHA GRANDE-RJ)
}

\section{BIOMETRICS OF FOREST FRAGMENTS SINUSIAE ARBOREAL DOMAIN TROPICAL ATLANTIC (ILHA GRANDE-RJ)}

\author{
Roberto Ricardo Rachid Saab Barbosa Cunha \\ Observatório Urbano/OUERJ/UN-Habitat \\ Gustavo Henrique de Souza Araujo \\ Ecoclean Ambiental \\ Manoel Gonçalves Rodrigues \\ Observatório Urbano/OUERJ/UN-Habitat - UNESA \\ Josimar Ribeiro de Almeida \\ Observatório Urbano/UERJ/UN-Habitat
}

\begin{abstract}
RESUMO
A agregação das plantas foi analisada através de quatro métodos de determinação do grau de agrupamento, chamados métodos de quadrados. A análise foi efetuada nos estratos inferior e superior, separadamente. O teste falhou para detectar distribuição agrupada apenas para UTF1 e L1, cujos valores de $\propto$ não foram significativamente diferentes de um. As demais morfoespécies, com exceção do grupo A2 (diversos gêneros) apresentamse fortemente agrupadas (significantes ao nível de 1\%). Quando foram consideradas todas as espécies que ocorreram na área, o índice encontrado também revelou distribuição fortemente agrupada. Efetuada a análise estatística para a escolha do modelo parabólico constatou-se que o modelo de equação $\log y=b o+b 1 c i+b 2 c^{2}$, estimava a área basal/ha com maior precisão para todos tipos florestais, exceto para a floresta (Tipo II), conforme mostra a Tabela 3. Para este tipo florestal, o modelo geral 01 ajustou às áreas basais, em relação aos DAPs, comparativamente aos demais modelos.A comparação entre os ajustamentos efetuados pelas funções BETA e MEYER, onde se evidencia o melhor ajustamento da função MEYER. Analisando-se os resíduos verificou-se que a função de MEYER subestimava, também, as frequências das árvores nas classes inferiores de DAPs, porém, abrangendo, apenas, as classes de 40 e $50 \mathrm{~cm}$, ou seja, numa amplitude menor que a abrangida pela função BETA.
\end{abstract}

Palavras-chave: Distribuição das árvores, Domínio tropical atlântico, Graus de agrupamento e Morfoespécie. 


\section{ABSTRACT}

The aggregation of the plants was analyzed by four different methods to determine the degree of clustering, called square methods. The analysis was performed in the lower strata and top separately. The test failed to detect grouped distribution only to UTF1 and L1, whose values were not significantly different from one. The other morphospecies, except for the A2 group (various genres) have strongly grouped (significant at the $1 \%$ level). When we considered all species occurring in the area, the index found also revealed strongly clustered distribution. Performed the statistical analysis for choosing the parabolic model found that the log equation model $y=$ bo ++ b1ci b2ci ${ }^{2}$, estimated basal area / ha more accurately for all forest types, except for the forest (Type II) as shown in Table 3. For this type of forest, the general model to the basal areas 01 set in relation to the DAPs compared to other modelos.A comparison between the adjustments made by the BETA functions and MEYER, where it shows the best-fit function MEYER. Analyzing the residues found that the MEYER function underestimated also the frequency of the trees in lower classes of DAPs, however, covering only the $50 \mathrm{~cm}$ and 40 class, i.e., a lower amplitude than that covered by BETA function

Keywords: Distribution of trees, the tropical Atlantic domain, Degrees grouping and morphospecies.

\section{INTRODUÇÃO}

A distribuição espacial das árvores no povoamento florestal é uma importante característica, O conhecimento da distribuição espacial de cada espécie, facilita os programas de aproveitamento como, também oferece informação para manejo, silvicultura, dendrologia e ecologia. A escolha do esquema de amostragem em inventários florestais, assim como o tamanho e forma das unidades de amostra, são influenciadas pelo tipo de distribuição das árvores no terreno (Loetsh 1973, Singh 1974). Considerando a dificuldade em obter-se uma amostra de indivíduos aleatórios para seus vizinhos mais próximos, Pielou (1959), desenvolveu um método que usa distâncias de pontos aleatórios para a planta mais próxima. Clark \& Evans (1954), preconizaram o método da distância de um indivíduo para o vizinho mais próximo, como uma medida para descrever o padrão de distribuição dos indivíduos de uma população.

A influência da distribuição espacial na precisão relativa dos sistemas de amostragem sistemática e aleatória foi investigada por Payandeh (1970, 1971 a. 1971 b). Seus estudos demonstraram que a amostragem sistemática é mais eficiente ou tão precisa quanto à amostragem aleatória, para populações florestais 
de distribuição espacial agrupada ou quase aleatória, quando a variável considerada foi a frequência de árvores por unidade de área. Jack (1961), investigando a distribuição espacial das árvores em floresta tropical africana, conclui que, considerando todas as espécies em conjunto, sua distribuição espacial não se ajusta à distribuição Normal ou de Poisson, mas está mais relacionada com uma distribuição agrupada, como a Binomial Negativa. Payandeh (1970) apresenta diversos métodos mais comuns para expressar a não-aleatoriedade na distribuição espacial de populações naturais. De uma maneira geral, os métodos são classificados em métodos de quadrados e métodos de distância.

A comparação entre os ajustamentos efetuados pelas funções BETA e MEYER, onde se evidencia o melhor ajustamento da função MEYER. Analisandose os resíduos verificou-se que a função de MEYER subestimava, também, as frequências das árvores nas classes inferiores de DAPs, porém, abrangendo, apenas, as classes de 40 e $50 \mathrm{~cm}$, ou seja, numa amplitude menor que a abrangida pela função BETA.

\section{MATERIAL E MÉTODOS}

Como método de distâncias de pontos aleatórios para a planta mais próxima, usou-se Pielou (1959). Como um índice de não-aleatoriedade, expressão $\propto=$ T.D. $\varpi$, onde $\propto=$ índice de não-aleatoriedade; $\pi=3.141593 ; \mathrm{D}=$ densidade, ou número de árvore por unidade de área, expressa na mesma unidade em que foram tomadas as distâncias; e $\varpi=$ média dos quadrados das distâncias de pontos aleatórios para as plantas mais próximas. Com esse índice, valores iguais a 1 indicam distribuição espacial aleatória; valores > 1 indicam distribuição espacial agrupada e aqueles $<1$ indicam distribuição espacial regular.

O teste de significância do afastamento de $\propto$ do valor unitário é feito pela distribuição do $\mathrm{X} 2(2 \mathrm{n} \propto$ segue aquela distribuição, com $2 \mathrm{n}$ graus de liberdade). A densidade pode ser obtida por amostragem independente aquela das medidas de distâncias, ou por enumeração completa. Neste trabalho adotou-se esta última alternativa. As distâncias foram medidas em um mapa na escala de 1:500 no qual foram localizadas todas as árvores levantadas no fragmento florestal. Para cada espécie estudada, foram alocados 100 pares de coordenadas aleatórias num total 
de 1.200 pares. A fim de prevenir que a planta mais próxima de determinado ponto aleatório pudesse se situar fora da área de estudo, estabeleceu-se uma borda de 10 metros. Todas as coordenadas situadas dentro dessa faixa foram rejeitadas.

A agregação das plantas foi analisada através de quatro métodos de determinação do grau de agrupamento, chamados métodos de quadrados. A análise foi efetuada nos estratos inferior e superior, separadamente. $O$ índice de MacGuinnes é I.G.A. $=\mathrm{D} / \mathrm{d}$ onde $\mathrm{D}=$ número total de plantas/número total de quadrados, $d=-\square$ 1-F/100 e $F=$ número de quadrados em que ocorre a espécie/número total de quadrados - 100 onde: I.G.A. = índice de grau de agregação, $D=$ densidade observada, $d$ = densidade esperada, $F=$ frequência $e$ $\square n$ $=$ logaritmo natural ou neperiano. Os valores de I.G.A. menores que 1 significam tendência a uma distribuição regular. Os valores de I.G.A. maiores que 2 indicam agregação. $O$ índice de Fracker \& Brischle é $K=(D-d) / d 2$, onde: $K=$ índice de agregação, $D=$ densidade observada, $d=$ densidade esperada; $D$ e $d$ são calculados como para o índice de MacGuinnes. Os valores de $\mathrm{K}$ menores que 0,15 indicam não agrupamento. Os valores entre 0,15 e 1,0 indicam tendência a se agrupar. Os valores maiores que 1,0 indicam agrupamento. $O$ índice de Payandeh é $\mathrm{P}=\mathrm{V} / \mathrm{M}$ onde: $\mathrm{P}$ = índice de agregação, $\mathrm{V}=$ variância do número de plantas por quadrado. $\mathrm{M}=$ média do número de plantas por quadrado. Os valores de $\mathrm{P}$ menores que 1,0 indicam a inexistência de agrupamento. Os valores de $P$ entre 1,0 e 1,5 indicam tendência a agrupamento e os valores maiores que 1,5 indicam agrupamento.

O índice de Hazen é $\mathrm{IH}=\mathrm{S} 2 / \bar{X}(\mathrm{n}-1)$, onde: $\mathrm{IH}=$ índice de agregação. S2 = variância, $\bar{X}=$ média do número de plantas por quadrado, $\mathrm{n}=$ número de observação. Os resultados do método de quadrados de Hazen são analisados através de Qui-quadrado $\left(\mathrm{X}^{2}\right)$. Os valores de IH menores que o valor de Quiquadrado a um nível de $75 \%$ de probabilidade significam não agrupamento da espécie e os valores de IH maiores que Qui-quadrado a $99 \%$ de probabilidade indicam agrupamento.

Para a quantificação dos estoques de madeira existentes nos diversos tipos de fragmentos florestais efetuou-se a cubagern em pé das árvores, utilizando-se 0 relascópio de Banda Larga. Posteriormente. Processou-se o cálculo dos volumes sobre casca, usando-se a fórmula de Smalian para tal objetivo. Os volumes 
calculados deram origem às equações volumétricas usadas para as estimativas do estoque. Para estimar os parâmetros K e B da função de Meyer, pela metodologia apresentada por Alexander \& Edminster (1977), estimando-se a Densidade em função dos coeficientes de uma equação parabólica.

A variável dependente representou a proporção de espaço ocupado pelas árvores por classe de área basal e, a independente às DAPs. Procurou-se, também, testar outros modelos, a fim de se obter maior precisão nas estimativas: y $=b o+b 1 . D A P+b 2$ DAP2; log $y=b o+b 1$. DAP + b2 DAP2; log y = bo + b1 log $\mathrm{DAP}+\mathrm{b} 2 \log$ DAP2 e 1/y = bo + b1 1/DAP + b2 1/DAP2.

O parâmetro B da equação de Meyer foi estimado em função da constante de Liocourt, conforme a seguinte expressão: $B=($ Inq)/W, onde: $q=$ constante de Liocourt, $W$ = intervalo de classe de DAP. O valor da constante $(q)$ foi calculado, usando-se o coeficiente (bl) de regressão linear estimada entre (In Ni) e os DAPs medidos, ou seja: $q$ = e-b1.W. substituindo-se a densidade original de Meyer K.eBci na expressão de Densidade proposta por Moser (1976), também citado por Alexander \& Edminster (1977), e, simplificando-se os termos obteve-se a constante K, ou seja:

$$
\text { Densidade }=\mathrm{K}=\sum_{i=1}^{n} \quad(\mathrm{bo}+\mathrm{b} 1 \mathrm{ci}+\mathrm{b} 2 \mathrm{ci} 2) \mathrm{e}-\mathrm{Bci}
$$

A Densidade, neste caso, foi estimada pela seguinte expressão: Densidade $=$ bo $\sum \mathrm{Ni}+\mathrm{b} 1 \sum \mathrm{Ni} \mathrm{ci}+\mathrm{b} 2 \sum \mathrm{Ni}$ ci2. Sendo bo, e b1 e b2 os coeficientes da regressão da equação parabólica ajustados por tipo florestal, e (c) as DAPs medidas.

\section{RESULTADOS E DISCUSSÃO}

Nas parcelas de $5 \mathrm{~m} \times 5 \mathrm{~m}$, na análise geral, as plantas mostraram apenas tendências a se agrupar (distribuição aleatória) e nas parcelas de $100 \mathrm{~m} \times 100 \mathrm{~m}$ apresentaram uma ocorrência totalmente agrupada (distribuição não aleatória). Este fato vem ratificar a afirmativa de Silva \& Lopes (1982) a respeito da influência nos resultados, causada pelo tamanho da parecla, quando são utilizados os "métodos de quadrados" na determinação do grau de agregação das plantas. 
Tabela 1 - Graus de agregação (G.A.) das plantas na Floresta de Dois Rios, em parcelas de tamanhos diferentes, sem considerar separação em estratos.

\begin{tabular}{|c|c|c|c|c|c|c|}
\hline \multirow[t]{2}{*}{ Método } & \multirow{2}{*}{$\begin{array}{l}\text { Frequência } \\
\text { (\%) }\end{array}$} & \multicolumn{2}{|c|}{ Densidade } & \multirow[t]{2}{*}{ Média } & \multirow[t]{2}{*}{ Variância } & \multirow[t]{2}{*}{ G.A. } \\
\hline & & Observada & Esperada & & & \\
\hline \multicolumn{7}{|c|}{ Parcelas de $5 \mathrm{~m} \times 5 \mathrm{~m}(0,0025 \mathrm{ha})$} \\
\hline MacGuinnes & 20,95 & 0,42 & 0,23 & - & - & 1,80 \\
\hline $\begin{array}{c}\text { Fracker \& } \\
\text { Brischle }\end{array}$ & 20,95 & 0,42 & 0,23 & - & - & 1,80 \\
\hline Payandeh & - & - & - & 0,42 & 0,56 & 1,30 \\
\hline Hazen & - & - & - & 0,42 & 0,56 & 780,63 \\
\hline \multicolumn{7}{|c|}{ Parcela de $100 \mathrm{~m} \times 100 \mathrm{~m}$ (1 ha) } \\
\hline MacGuinnes & 91,43 & 2,46 & 66,31 & - & - & 27,00 \\
\hline $\begin{array}{l}\text { Fracker \& } \\
\text { Brischle }\end{array}$ & 91,43 & 2,46 & 66,31 & - & - & 10,00 \\
\hline Payandeh & - & - & - & 66,31 & $8.642,93$ & 130,00 \\
\hline Hazen & - & - & - & 66,31 & $8.642,93$ & $4.431,00$ \\
\hline
\end{tabular}

A Tabela 2 mostra os valores do índice de não-aleatoriedade encontrados para as morfoespécies estudadas. A significância de $\alpha$ foi comparada na tabela dos limites de significância calculados por Pielou ( 1959).

Tabela 2 - Índices de não-aleatoriedade das espécies estudadas.

\begin{tabular}{cccc}
\hline Morfoespécie & Frequência & Densidade por $\mathrm{m}^{2}$ & $\alpha$ \\
\hline A1 & 419 & $1,19 \times 10^{-3}$ & $1,77^{\mathrm{b}}$ \\
$\mu 1$ & 426 & $1,23 \times 10^{-3}$ & $1,40^{\mathrm{b}}$ \\
T1 & 165 & $4,80 \times 10^{-4}$ & $3,24^{\mathrm{b}}$ \\
U $\mu$ & 183 & $5,29 \times 10^{-4}$ & $1,87^{\mathrm{b}}$ \\
TV1 & 129 & $3,51 \times 10^{-4}$ & $1,51^{\mathrm{b}}$ \\
A2 & 422 & $1,19 \times 10^{-3}$ & $1,23^{\mathrm{a}}$ \\
B1 & 181 & $5,11 \times 10^{-4}$ & $2,37^{\mathrm{b}}$ \\
N2 & 103 & $2,86 \times 10^{-4}$ & $1,48^{\mathrm{b}}$ \\
UTF1 & 185 & $5,11 \times 10^{-4}$ & 1,00 \\
U3 & 1542 & $3,85 \times 10^{-4}$ & $1,29^{\mathrm{b}}$ \\
L3 & 274 & $7,77 \times 10^{-4}$ & 1,19 \\
Demais & 4153 & $1,19 \times 10^{-2}$ & $1,43^{\mathrm{b}}$ \\
Morfoespécies & & &
\end{tabular}

a. Significante ao nível de $5 \%$.

b. Significante ao nível de $1 \%$.

O teste falhou para detectar distribuição agrupada apenas para UTF1 e L1, cujos valores de $\propto$ não foram significativamente diferentes de um. As demais morfoespécies, com exceção do grupo A2 (diversos gêneros) apresentam-se 
fortemente agrupadas (significantes ao nível de 1\%). Quando foram consideradas todas as espécies que ocorreram na área, o índice encontrado também revelou distribuição fortemente agrupada.

Quando a distribuição espacial é agrupada, unidades de amostra retangulares são mais precisas, pois tendem a absorver melhor os efeitos das "clareiras" - espaços vazios, sem a presença de árvores -, que as unidades quadradas ou circulares. Pesquisas sobre a eficiência do processo de amostragem aleatória versus a amostragem sistemática devem ser conduzidas na floresta tropical úmida, pois, conforme demonstra Payandeh (1971 a), em distribuições agrupadas o processo sistemático é mais eficiente.

Efetuada a análise estatística para a escolha do modelo parabólico constatou-se que o modelo de equação log $y=b o+b 1 c i+b 2$ ci2, estimava a área basal/ha com maior precisão para todos tipos florestais, exceto para a floresta (Tipo II), conforme mostra a Tabela 3. Para este tipo florestal, o modelo geral 01 ajustou às áreas basais, em relação aos DAPs, comparativamente aos demais modelos (Hosokawa et al, (1975)).

Utilizando-se os coeficientes de regressão da Tabela 3, efetuou-se os cálculos das constantes da função de Meyer por tipo florestal através das equações $\mathrm{B}, \mathrm{G}, \mathrm{K}$ e densidade, conforme mostra a Tabela 2, necessárias para se balancear as distribuições de frequências das florestas em estudo, a fim de se ter ideia do estoque e efetuar previsões de corte.

Tabela 3 - Estimativa das equações de regressão usadas para o cálculo dos parâmetros da função de MEYER.

\begin{tabular}{c|c|c|c|c|c|c|c}
\hline \multicolumn{7}{c}{ TIPO FLORESTAL } & \\
\hline- & I & II & III & IV & V & VI & VII \\
\hline bo & 0,43611 & 0,04406 & 3,28980 & 0,27919 & 0,51286 & 0,75535 & 0,16331 \\
b1 & $-0,00058$ & 0,00487 & $-0,03295$ & 0,00378 & $-0,00076$ & $-0,00919$ & 0,00521 \\
b2 & $-0,00001$ & $-0,00003$ & 0,00009 & $-0,00004$ & $-0,00002$ & 0,00002 & $-0,00004$ \\
$\mathrm{R}^{2} \mathrm{Aj}$ & 0,84066 & 0,89257 & 0,84751 & 0,96257 & 0,75604 & 0,96651 & 0,96651 \\
$\hat{S} \hat{y} x$ & 0,10462 & 0,07292 & 0,26435 & 0,12678 & 0,06916 & 0,16157 & 0,05433 \\
IF & 0,30410 & 0,20406 & - & 0,32411 & 0,16955 & 0,30378 & 0,15109 \\
\hline
\end{tabular}

A comparação entre os ajustamentos efetuados pelas funções BETA e MEYER, onde se evidencia o melhor ajustamento da função MEYER. Analisando- 
se os resíduos verificou-se que a função de MEYER subestimava, também, as frequências das árvores nas classes inferiores de DAPs, porém, abrangendo, apenas, as classes de 40 e $50 \mathrm{~cm}$, ou seja, numa amplitude menor que a abrangida pela função BETA.

As maiores subestimativas foram encontradas na classe de $40 \mathrm{~cm}$, nos tipos florestais I, V e VI, cujas diferenças residuais $21.27 \%, 19,41 \%$ e 22,37\%, respectivamente. Valores estes menores que aqueles estimados pela função BETA nas mesmas classes. Entre 60 e $110 \mathrm{~cm}$ ocorrem superestimativas das frequências com valores, ligeiramente, superiores às estimativas da função BETA.

Entretanto, para todos os tipos florestais, ao contrário da função BETA, a função MEYER estimou com precisão as frequências das árvores nas classes superiores de DAPs. Com base nos dados medidos em cada unidade amostral, e, na cubagem individual das árvores em pé, selecionou-se as seguintes equações usadas para as estimativas volumétricas das espécies florestais nos diversos tipos de vegetação inventariados (Tabela 5).

Tabela 4. - Constantes da função de MEYER por tipo florestal.

\begin{tabular}{|c|c|c|c|c|c|c|c|}
\hline \multicolumn{7}{|c|}{ TIPO FLORESTAL } \\
\hline- & I & II & III & IV & V & VI & VII \\
\hline q & 1,2814 & 1,2587 & 1,3977 & 1,3869 & 1,3797 & 1,3437 & 1,3391 \\
\hline B & 0,0248 & 0,02301 & 0,03348 & 0,03271 & 0,03219 & 0,02954 & 0,02920 \\
\hline K & 379,292 & 236,356 & 488,505 & 594,603 & 614,993 & 465,792 & 456,287 \\
\hline Densidade & 203,900 & 93,564 & 720,376 & 190,059 & 231,825 & 139,792 & 163,470 \\
\hline
\end{tabular}

Tabela 5 - Estimativa das equações hipsométricas para os tipos de fragmentos florestais.

\begin{tabular}{|c|c|c|c|c|c|c|}
\hline \multirow{2}{*}{ Modelo } & \multicolumn{4}{|c|}{ Coeficientes } & \multirow{2}{*}{$\mathrm{R}^{2} \mathrm{Aj}$} & \multirow{2}{*}{$\hat{y x}$} \\
\cline { 2 - 5 } & $\mathrm{bo}$ & $\mathrm{b} 1$ & $\mathrm{~b} 2$ & $\mathrm{~b} 3$ & & 3,4721 \\
\hline $\begin{array}{c}\mathrm{H}=\mathrm{bo}+\mathrm{b} 1 \mathrm{~d}+ \\
\mathrm{b} 2 \mathrm{~d}^{2}+\mathrm{b} 3 \mathrm{~d}^{3}\end{array}$ & 1,78898 & 0,064889 & $-0,55538$ & 0,00001 & 0,75253 & \\
\hline $\begin{array}{c}\mathrm{hc}=\mathrm{bo}+\mathrm{b} 1 \mathrm{~d}+ \\
\mathrm{b} 2 \mathrm{~d}^{2}+\mathrm{b} 3 \mathrm{~d}^{3}\end{array}$ & 0,88674 & 0,031647 & $-0,00798$ & 0,00002 & 0,51961 & 2,8358 \\
\hline $\begin{array}{c}\text { Logv }=\text { bo }+\mathrm{b} 1 \\
\text { logd }+\mathrm{b} 2 \text { logc }\end{array}$ & $-3,95296$ & 2,04759 & 0,63482 & - & 0,96667 & 0,12319 \\
\hline
\end{tabular}

$\mathrm{d}=$ DAP $\mathrm{cm} ; \mathrm{hc}=$ Altura $(\mathrm{m}) ; \mathrm{IF}=0,3036$.

\section{REFERÊNCIAS BIBLIOGRÁFICAS}

ALEXANDER. R.R. \& EDMINSTER, C.B. Uneven - aged management of old growth 
Spruce - Fir forest: Cutting methods and stano structure goals for the initial entry. USDA Forest Service, 1977, 12p.

CLARK, P.J. \& EVANS, F.C. Distance to nearest neighbor as a measure of' spatial relationships in populations. Ecology, 35 (4): 445-53,1954.

JACK, W.H. The spatial distribution of' sterns in a tropical high forest. Emp. For. Ver., 40: 234-41, 1961.

LOETSCH, F.; ZOEHRER, F. \& HALLER, K. E. Forest Inventory, Berlin, 1975. $469 p$.

LOETSCH, F.; ZOHRER. F.; HALLER, K.E. Forest inventorv, Munich. BLV. 1973. V. 2.

MEYER, H. A. Structure, growth and drain in balanced uneven-aged forest. Journ. For., 50 (2): 85-92. 1952.

MOSER, J .W. Specificarion of density for the inverse J-Shaped diameter distribution. For. Sci. (52): 744-47. 1976.

PAYANDEH, B. Spatial patterm of trees in the major forest types of northern Ontario. Can. J. For. Res. 4: 8-14. 1974.

PAYANDEH, B. \& EK. A.R. Observation on spatial distribution and the relative precision of systematic sampling. Can. J. For. Res. 1: 216-22. 1971 a.

PAYANDEH, B. \& PAINE, B.P. Relative precision of' two-dimensional systematic sampling as a function of non-randommes index. Can. J. For. Res. 1: 167-73. $1971 b$.

PIELOU, E.C. The use of point to plant distances in study of pattern of plant populations. Journal of Ecology, 47: (3): 607-3,1959.

SING. K.D. Spatial variation patterns in the tropical rain forest. Unasylva, 26 (106): 18-23, 1974.

Recebido: 25/01/2015

Aprovado: 10/06/2015 